\section{Duração da gestação em relação à idade de éguas da raça Puro Sangue de Corrida, aos pesos do potro e da placenta, e ao horário do parto}

\section{Gestation length related to the age in Thoroughbred mares, to placenta's and newborn foal's weight and parturition time}

\author{
Mario KUR'YZ FILHO'; Neiva Medianeira DEPRÁ²; Joaquin Lopes de ALDA²; Inês Nicoloso \\ de CASTRO ${ }^{3}$; Flávio Desessards DE LA CORTE*; José Henrique Souza da SILVA; ; Carlos \\ Antonio Mondino SILVA ${ }^{4}$
}

\begin{abstract}
CORREPONDENCE TO:
Mario Kurtz Filho

Departamento de Morfologia, CCS.

Universidade Federal de Santa Maria 97119-900. Santa Maria - RS - Brasit e-mail:kurtz@saigon.cpd.utsm.br

1 - Departamento de

Morfologia, CCS, Universidade

Federal de Santa Maria - RS

2 - Médico velerinário, Haras

Santa Maria de Araras

Sāo José dos Pinhais - PA

3 - Médico veterinario, Haras

Santa Maria de Araras

Bajé - RS

4. Departamento de Clínica de Grandes Animais, CCR,

Universidade Federal de Santa Maria - RS

5 - Departamento de Zootecnia, CCR, Universidade Federal de

Santa Maria - RS
\end{abstract}

\title{
RESUMO
}

Este estudo foi realizado com o propósito de determinar parâmetros fisiológicos relativos ao pós-parto e puerpério na égua e potro. De 01/07/1983 a 25/11/1992 foram observados 390 partos em éguas da raça Puro Sangue de Corrida. Éguas mais velhas produziram potros e placentas mais pesados; a idade materna não influenciou na duração da gestação e esta última não esteve relacionada com o período de concepção ou com o peso do produto. Éguas mais velhas, com endométrio sadio, apresentaram placentas mais pesadas que éguas jovens, também saudáveis. $87,54 \%$ dos partos ocorreram à noite (entre 18 horas e 7 horas) relacionando-se com períodos de tranqüilidade ambiental.

UNITERMOS: Éguas; Potros; Animais recém-nascidos; Placenta; Parto; Período pós-parto.

\section{INTRODUÇÃO}

$\mathrm{O}$ periodo de gestaçāo médio ná égua Puro Sangue de Corrida e de 338-340 dias. com uma variação de 310-374 dias (Whitwell: Jeefcott"1, 1975; Ginther ${ }^{4}, 1979$; Rossdale; Ricketts ${ }^{17}$, 1980)). Ropihat et all. ${ }^{15}(1969)$, Campitelli et al. $2(1982-1983)$ e Hodge e' al." (1982) observaram que a época do ano e o fotoperiodo podem influenciar a duração da gestação.

O tamanho do potro ao nascer varia amplamente, de acorclo com o tipo de eqüino. Nos animais de tamanho médio. o peso relativo do potro é ao redor de $10 \%$ do peso da égua. Existe uma correlação entre o peso ao nascimento e a idade materna, mas as bases fisiológicas sāo incertas; a progressiva degeneraçāo do endométrio com o avanço da idade, especialmente após os 12 anos de idade, poderia ser responsabilizada por isso. Tamanho fetal c idade gestacional parecem estar intimamente relacionados (Platt ${ }^{12}, 1984$ ).

A manifestaçāo externa das características peso e altura nāo sāo evidentes nos primeiros meses de vida. porém o são ao redor do primeiro ano de idade; ou seja, há uma tendência de as caracteristicas relacionadas a tamanho e peso tornarem-se mais evidentes com o aumento da idade (Ilintz et al.5, 1978; Hintz et al. . 1979b).

Rossdale; Short ${ }^{1 "}(1967)$ e Jeffeot" (1972) verificaram uma concentraçāo de partos entre as 19 e 7 horas, com máxima incidencia entre as 20 c 24 horals.

Com o objetivo de estabelecer parâmetros fisiológicos en nosso meio para os tópicos descritos foi desenvolvido este trabalho.

\section{MATERIAL E MÉTODO}

Foram observados 390 partos cm eqüinos da raça Puro Sangue de Corrida, alojados no Haras Santa Maria de Araras, São José dos Pinhais (253!' de latitude sul e 49"10' de longitude oeste), Paraná, no período compreendido entre 1" de jullho de 1983 a 25 de novembro de 1992.

As seguintes variávcis foram consideradas:

1. Idade materna: $\mathrm{cm}$ anos

2. Idade gestacional: período compreendido entre a ovulaçáo e a expulsão completa do potro. em dias

3. Hora do parto

4. Peso da placenta $(\mathrm{kg})$

5. Peso dos potros $(\mathrm{kg})$

Para registro dos dados foi utilizada uma ficha obstétrica, a qual, durante o período de colheita dos dados. foi ampliada, sendo por isto inconstante o número de observações entre variáveis. Observe-se que o mancjo e o nível nutricional a que as éguas estavam submetidas foram elevatdos e constantes e que potros com qualquer tipo de problema, comportamento alterado, ou que tenham sido submetidos a aleitamento artificial ou outra intervençāo qualquer, foram desconsiderados. Da mesma forma, éguas e placenlas com qualquer tipo de alteraçăo foram descartadas para este estudo. Nāo foram consideradas características individuais de cada égua que pudessem influir sobre a duração da gestação.

Os dados foram submetidos a análise de correlaçẫo e regressão, análise de variância e Teste F (Steel; Torric ${ }^{29}$, 1980). 


\section{RESULTADOS}

A idade média das éguas foi de 9,4 anos, com amplitude de 4 a 20 anos. O período gestacional médio foi de 334 dias, com uma variação de 315 a 360 dias. Nảo foi observada relação significativa entre mês de concepçăo (amplitude: agosto e dezembro) e período gestacional $(p<0,21 ; r=0,(02)$.

Os períodos de gestaçāo, os pesos das placentas e os pesos dos potros. em 3 grupos de éguas divididas nas faixas etárias de 4 a 5 anos, 6 a 12 anos e 13 a 20 anos, são apresentados na Táb. 1 . A duração da gestação no grupo de 4 a 5 anos teve a média de 332,1 dias; no grupo de 6 a 12 anos foi de 333,6 dias e no de 13 a 20 anos, de 338,2 dias.
Nāo houve relação entre a duraçāo da gestação e o peso do produto an nascer. Não houve relaçāo entre idade das éguas e idade gestacional.

O peso médio da placenta no grupo de 4 a 5 anos foi de $5,35 \mathrm{~kg}$, no de 6 a 12 anos foi de $5,81 \mathrm{~kg}$ e no de 13 a 20 anos foi de $6,33 \mathrm{~kg}$. $\mathrm{O}$ peso médio dos potros no grupo de 4 a 5 anos foi de 44,9, no de 6 a 12 anos foi de $51,5 \mathrm{~kg}$ e no de 13 a 20 anos foi de $53,6 \mathrm{~kg}$. Observou-se que o grupo das éguas de maior idade ( 13 a 20 anos) foi o que apresentou placentas mais pesadas $(\mathrm{p}<0,0(12)$. O grupo mais jovem (4 a 5 anos) produziu os produtos mais leves $(p>0,00() 2)$.

Quanto à hora de ocorrência dos partos, verificou-se que 281 deles, correspondentes a $87,54 \%$, ocorreram entre as 18 e 7 horas, 24 $(7,48 \%)$ entre as $7 \mathrm{e} 12$ horas; $16(4,98 \%)$ entre as 12 e 18 horas.

Tabela 1

Médias da duraçāo da gestaçāo (DG); peso médio das placentas (PPL) e dos potros (PP), relacionados a três grupos etários de éguas, em 362 partos (1 78 machos, 184 fêmeas) assistidos no período de $1^{\circ}$ de julho de 1983 a 25 de novembro de 1992, no Haras Santa Maria de Araras, Sāo José dos Pinhais, PR.

\begin{tabular}{|c|c|c|c|c|c|c|c|c|c|}
\hline \multirow{2}{*}{ Parâmetro } & \multicolumn{3}{|c|}{ Idade: 4-5 anos } & \multicolumn{3}{|c|}{ Idade: 6-12 anos } & \multicolumn{3}{|c|}{ Idade: $13-20$ anos } \\
\hline & $\mathrm{n}$ & $x$ & c & $\mathrm{n}$ & $x$ & 4 & $\mathrm{n}$ & $x$ & $d$ \\
\hline DG (dias) & 07 & $332,10^{a}$ & $\pm 10,00$ & 82 & $333,60^{a}$ & $\pm 7,80$ & 17 & $338,20^{a}$ & $\pm 9,99$ \\
\hline PPL (kg) & 24 & $5,35^{b}$ & $\pm 0,90$ & 256 & $5,81^{b}$ & $\pm 1,28$ & 59 & $6,33^{a}$ & $\pm 1,34$ \\
\hline $\mathrm{PP}(\mathrm{kg})$ & 14 & $44,90^{\circ}$ & $\pm 5,55$ & 198 & $51,50^{\mathrm{a}}$ & $\pm 6,74$ & 51 & $53,60^{a}$ & $\pm 7,05$ \\
\hline
\end{tabular}

Na mesma linha, médias seguidas de letras diferentes diferem significativamente pelo Teste de Tukey.

figura 1

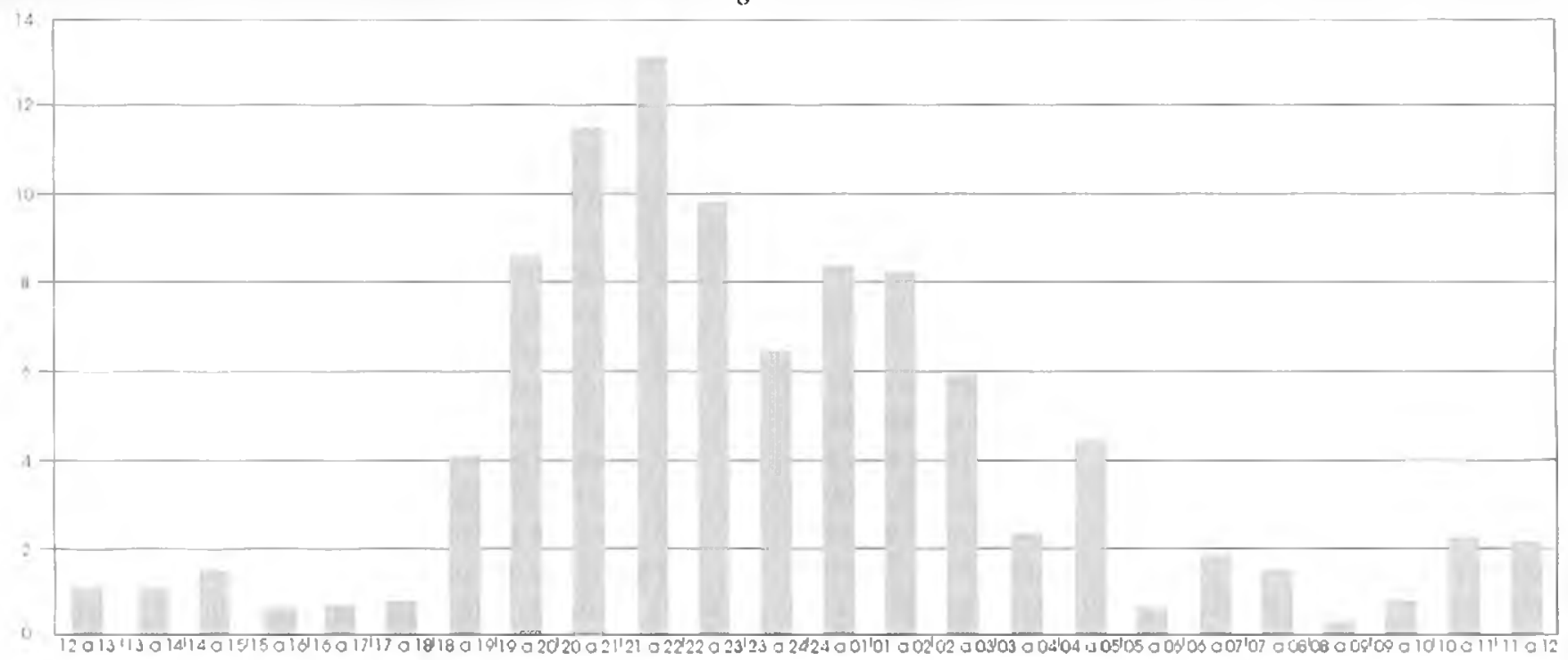

Freqüência de distribuiçāo dos horários de parto. Os intervalos de classe com longitude de 1 hora estāo representados no eixo horizontal. Observaçóes em 321 partos no periodo de $1^{\circ}$ de julho de 1983 a 25 de novembro de 1992. São José dos Pinhais, PR.

\section{DISCUSSĀO}

O período gestacional médio encontrado neste estudo foi de 334 dias, com amplitude de 315 a 360 dias. Como não foram consideradas características individuais de cada égua, o número de partos observados foi suficiente para diluir ou anular possíveis influências. Este período gestacional concorda com os observados por Whitwell; Jeffeott ${ }^{21}$ (1975), Ginther ${ }^{4}$ (1979), Rossdale; Ricketts $^{17}(1980)$.
Não se verificou diferença na duração da gestação nas diferentes épocas de parto; ou seja, concepçōes ocorridas em agosto ou dezembro não apresentaram diferenças nos seus períodos gestacionais; esses resultados assemelham-se aos de Jeffeott ${ }^{9}$ (1972) trabalhando com pôneis. Isso indica que, em nossas condiçōes de latitude, o fotoperíodo não influiu na duração da gestaçāo. Observaçōes feitas por Ropiha et al. ${ }^{15}$ (1969), na Austrália, numa latitude maior, indicam que concepçōes ocorridas no início da primavera resultaram em gestações mais longas 
do que as de qualquer outro mês; Hintz et al. ${ }^{6}$ (1979 a) têm a mesma conclusão. Como o fotoperíodo varia na razão direta da latitude, a duraçāo da gestação deveria sofrer uma variaçāo cada vez maior na medida em que as éguas se afastassem do equador.

Não houve relação entre duração da gestação e peso do produto ao nascer, ou seja, gestaçôes mais curtas não significaram produtos mais leves e gestações longas não produziram produtos mais pesados. Potros com período gestacional maior ou menor do que a média podem ter tamanho e peso médios. A aparência clínica do potro e a duração da gestação devem ser elementos conjuntos de avaliação. Aqui se observou que a duração da gestação varia consideravelmente e que tanto o menor periodo gestacional (315 dias) como o maior (360 dias) resultaram em potros normais a termo. A maturidade fetal pode ser influenciada e desencadeada por fatores de sobrecarga orgânica (estresse fetal, placentite, por exemplo) c parece estar intimamente relacionada ao aumento das concentraçōes de Ca, Na e K do colostro (Rose et al. "', 1982; LeBlanc"1, 1990).

Com o aumento da idade, as éguas tendem a produzir produtos mais pesados e placentas, conseqüentemente, mais pesadas. Embora mais velhas, éguas com até 20 anos conseguiram providenciar um ambiente intra-uterino adequado para um ótimo desenvolvimento do feto. Estes resultados diferem dos de Campitelli et al. ${ }^{2}$ (1982-83), que observaram animais da mesma raça dos aqui estudados, embora não tenham comentado o nível nutricional e o manejo. Verifica-se também que as éguas com idade entre 6 e 20 anos pariram produtos mais pesados do que aquelas com idade entre 4 e 5 anos. Aparentemente, este resultado sugere uma provável relaçāo com o tamanho do útero, que possuiria a capacidade de determinar o tamanho do feto. PoolAnderson et al. ${ }^{13}$ (1994) também verificaram que multíparas pariram potros mais pesados do que primiparas. Chevalier; Palmer' (1982) observaram que a vesícula embrionária de éguas primíparas era menor do que em éguas maduras, provavelmente em virtude do útero menor. Esses autores observaram ainda que embriōes de raças pesadas eram maiores que os de raças mais leves.

Não houve influência da idade das éguas na duraçāo da gestaçāo; a idade da māe. portanto, parece ter pouca importância prática na determinaçāo do período gestacional. Hintz et al. ${ }^{6}$ (1979a) observaram 1.704 éguas da mesma raça e também não verificaram relaçāo alguma da idade das éguas com a duração da gestação.

Observando-se a distribuição dos horários dos partos ao longo do dia (Fig. 1), tem-se que $87,54 \%$ dos partos ocorreram no período compreendido entre as 18 e 7 horas e $12,46 \%$ entre as 7 e 18 horas. Os partos ocorreram nos períodos de maior tranqüilidade ambiental. Esta ocorrência pode ser reforçada pelo fato de que a maioria dos partos ocorreu nos meses de julho, agosto e setembro (74,15\%), quando amanhece às 7 horas e anoitece às 18 horas, além da rotina de trabalho no local encerrar-se às 17:30 horas e reiniciar-se às 7:30 horas. Estes achados corroboram aqueles citados por Rossdale; Short ${ }^{18}$ (1967); B.F. Trum apud Roberts (1971); Jeffcott' (1972); Bain; Howey ${ }^{1}$ (1975). Verga et al. ${ }^{20}$ (1982) sugerem que este período circadiano pode ser resultado da adaptaçāo evolutiva. Embora este seja o período de maior atividade de predadores, é também o período que maiores oportunidades oferece para o individuo ocultar-se. Isto pôde ser comprovado entre outras espécies: diferentes populações humanas foram analisadas, observando-se que aquelas que utilizam o período noturno para descanso concentram os partos entre as 3 e 4 horas. Em populações com atividades diurnas e noturnas, o horário de parto apresentou uma distribuição similar ao longo do dia (Kaiser; Halberg'", 1962).

\section{CONCLUSŌES}

A duração da gestação não é influenciada pela época da concepção ou pela idade dágua e nāo tem influência no peso do potro. A grande maioria dos partos $(87,54 \%)$ ocorre à noite (entre as 18 e 7 horas), em períodos de tranqüilidade ambiental. Éguas mais velhas, com idade entre 13 a 20 anos, produzem produtos sadios e, desde que nāo tenham alterada a estrutura e funçāo endometrial, produzem potros tão pesados quanto éguas de 6 a 12 anos. Éguas com idade entre 6 e 20 anos parem produtos mais pesados do que éguas com idade entre 4 e 5 anos. A placenta pesa ao redor de $11,5 \%$ do peso do produto.

\section{SUMMARY}

This study was performed with the aim of determining physiologic parameters regarding post foaling behavior in the mare, puerperium and foal. Three hundred and ninety deliveries of Thoroughbred mares were observed between July, 1983 and November, 1992 . Older mares produced heavier foals and placentae. The mare's age had no influence on gestational length, and this parameter was not related to the moment of conception or to the foal's weight. Older mares, with a healthy endometrium, eliminated heavier placentae compared to younger, healthy mares. Most of deliveries (87.54\%) occurred at night (between 6:00 p.m. and 7:00 a.m.), when environmental activities were at a minimum.

UNITERMS: Mares; Newborn animals; Foals; Parturition; Placenta; Postpartum period.

\section{REFERÊNCLAS BIBLIOGRÁFICAS}

1-BAIN, A.M.; HOWEY, W.P. Observation on the time of foaling in Thoroughbred mares in Australia. Journal of Repmduction and Fertility. p.545-6, 1975. Suplemento 23.

2-CAMPITELLI, S.; CARENZI. C.; VERGA, M. Factors which influence parturition in the mare and development of the foal. Applied Animal
Ethology, v.9, p.7-14, 1982-83.

3-CHEVALIER, F,; PALMER, E. Ultrasonic echography in the mare. Journal of Reproduction Fertility, p.423-30, 1982. Suplemento 32.

4-GINTHER, O.J. Reproductive biology of the mare. Ann Arbor,

McNaughton and Gunn, 1979. cap. 2; Parturition, postpartum period, and prepuberal period: p.359.

5-HINTZ, R.L.; HINTZ, H.F.; van VLECK, L.D. Estimation of heritabilities 
for weight. height, and front cannon bone circumference of Thoroughbred. Journal of Animal Science. v.47, n.6, p.1243-5, 1978.

6-HINTZ H.F.; HINTZ, R.L.; LEIN, D.H.; van VLECK, L.D. Length of gestation periods in Thoroughbred mares. Journal of Equine Medicine and Surgery, v.3, n.6, p.289-92, 1979a.

7-HINTZ, H.F.; HINTZ, R.L.; van VLECK, L.D. Growth rate of Thoroughbreds, effect of age of dame, year and month of birth, and sex of foal. Journal of Animal Science, v.48, n.3, p.480-7, 1979 b.

8-HODGE, S.L.; KREIDER, J.L.; POTTER. G.D.; HARMS. P.G.; FLEEGER. J.L. Influence of photoperiod on the pregnant and postpartum mare. American Journal of Veterinary Research, v.43, n.10, p.1752-5, 1982.

9-JEFFCOTT, L.B. Observation on parturition in crossbred pony mares. Equine Veterinary Journal. v.4, n.4, p.209-13. 1972.

10-KAISER, I.H.; HALBERG, F. Circadian aspects of birth. Annals New York Academy Sciences, v.98, p.1056-68, 1962.

11-LeBLANC, M.M. Induction of parturition in the mare: assessment of readiness for birth. In: KOTERBA, A.M.; DRUMMOND, W.H., KOSH, P.C. Equine clinical neonatology. Philadelphia. Lea \& Febiger, 1990. cap.3, p.34-9.

12-PLATT, H. Growth of the equine foetus. Equine Veterinary Journal. v.16, n.4, p. 247-52, 1984.

13-POOL-ANDERSON, K.; RAUB, R.H.; WARREN, J.A. Maternal influences on growth and development of full-sibbling foals. Journal of Animal Science, v.72, n.7, p.1661-6, 1994.

14-ROBERTS, S.J. Veterinary obstetrics and genital diseases. 2. ed. Ann Arbor, Edward Brothers, 1971. 776p.

15-ROPIHA. R.T.; MATTHEWS, R.G.; BUTTERFIELD, R.M.; MOSS, F.P;; McFADDEN, W.J. The duration of pregnancy in Thoroughbred mares. Veterinary Record, v.84, n.22, p.552-5, 1969.

16-ROSE, R.J.; ROSSDALE, P.D.; LEADON, D.P. Blood gas acid-base status in spontaneously delivered term-induced and induced premature foals. Journal of Reproduction and Fertility, p.521-8, 1982. Suplemento 32.

17-ROSSDALE, P.D.; RICKETTS, S.W. Equine stud farm medicine. London, Bailliere Tindall, 1980. p.564.

18-ROSSDALE, P.D.; SHORT. R.V. The time of foaling of Thoroughbred mares. Journal of Reproduction Fertility, v.13, p.341-3, 1967.

19-STEEL, R.G.D.; TORRIE. J.H. Principles and procedures of statistics, a biometrical approach. 2. ed. New York, McGraw-Hill, 1980.633p.

20-VERGA. M.; OBEROSLER. R.; SOMMARIVA. E.; FAZZINI, V. Analisi del comportimento perinatale in cavalli di razze diverse. Atti Della Societả Italiana Delle Scienze Veterinarie, v.36, p.212-5, 1982.

21-WHITWELL. K.E.; JEFFCOTT, L.B. Morphological studies on the fetal membranes of the normal singleton foal at term. Research in Veterinary Science. v.19, n.1, p.44-55, 1975. 